\title{
Implications of the growing incidence of global colorectal cancer
}

\author{
Benjamin W. Vabi ${ }^{1}$, John F. Gibbs ${ }^{2}$, Glenn S. Parker ${ }^{2}$ \\ ${ }^{1}$ Department of Surgery, WellSpan York Hospital, York, PA, USA; ${ }^{2}$ Department of Surgery, Hackensack Meridian School of Medicine at Seton Hall \\ University, Nutley, NJ, USA \\ Contributions: (I) Conception and design: JF Gibbs, BW Vabi; (II) Administrative support: None; (III) Provision of study materials or patients: None; \\ (IV) Collection and assembly of data: All authors; (V) Data analysis and interpretation: All authors; (VI) Manuscript writing: All authors; (VII) Final \\ approval of manuscript: All authors. \\ Correspondence to: Glenn Parker, MD, FACS, FASCRS. Jersey Shore University Medical Center, Department of Surgery, Ackerman-4, 1945 State \\ Highway 33, Neptune, NJ 07753, Email: eginvest7@aol.com.
}

\begin{abstract}
The expanding worldwide burden of colorectal cancer (CRC) is a significant public health issue. Understanding the shift in the geo-demographic, socioeconomic, environmental, and biogenetic distribution of CRC is paramount. The Human Development Index (HDI) measuring life expectancy, education, and gross national income is a composite index comparing health outcomes between countries. This has been shown to be a useful comparison tool in measuring the health dimension among high, middle, and low-income countries. CRC has a wide global distribution in incidence and mortality with majority of cases occurring in countries with a high or very high HDI. However, in developing countries and in those undergoing rapid socioeconomic growth, there has also been a marked rise in CRC rates as well. This pattern is noted globally and seems to correlate with increase in a country's specific HDI. Additionally, another unique pattern of CRC incidence has emerged with more cancers being diagnosed in adults younger than 50 years old. Further investigation is needed to determine CRC risks reduction and implementation of primary prevention and early detection strategies within different country specific healthcare systems. Globally, improvement in healthcare equality, access to medical care and screening for CRC particularly in resource-limited (low HDI) countries is essential.
\end{abstract}

Keywords: Colorectal cancer (CRC); global; Human Development Index (HDI)

Submitted Jan 29, 2020. Accepted for publication Jul 17, 2020.

doi: 10.21037/jgo-2019-gi-06

View this article at: http://dx.doi.org/10.21037/jgo-2019-gi-06

\section{Introduction}

According to the American Institute for Cancer Research, colorectal cancer (CRC) is the third most commonly diagnosed cancer in men and the second most CRC in women worldwide. In 2018 alone, approximately 1.8 million new cases of CRC were diagnosed with over 145,600 new cases occurring in the United States. Globally, the distribution of CRC burden varies widely (1). The Human Development Index (HDI) is a statistical tool to measure a country's overall achievement in its environmental and socioeconomic growth (2). It a snapshot of human development: a long and healthy life, being knowledgeable and having a decent standard of living. A long and healthy life expectancy is measured from birth (Life Expectancy Index). Knowledge is determined by the indicators of expected and mean years of schooling (Education Index). Finally, a decent standard of living is determined by the gross national index (GNI) per capital (3). The HDI is categorized as very high (mean $0.88 ; 0.80-0.95$ ), high (mean $0.76 ; 0.71-0.78$ ), medium (mean $0.61 ; 0.56-0.70$ ), or low (mean $0.53 ; 0.38-0.55$ ) (4). The United States (HDI 0.92) trends behind many other western countries with the $9^{\text {th }}$ highest incidence of CRC at 38.6 per 100,000 populations with the highest incidence occurring in Hungary and South Korea (5) (Table 1). Strikingly, cancerrelated death is expected to increase by $60 \%$ by 2030 (Figure 1). Understanding the paradigm shift in the geo- 
demographic, socioeconomic, environmental, biogenetic distribution of CRC and the factors affecting early detection and management is paramount to identifying polices and interventions to eradicating it. The purpose of this expanded review is to address the trends and implications of CRC globally using their HDI. Ultimately, country specific resource allocation in formulating strategies in early CRC detection is fundamental in decreasing the global rise in

Table 1 Global colorectal cancer rates: both sexes

\begin{tabular}{lcc}
\hline Rank & Country & Age-standardized rate per 100,000 \\
\hline 1 & Hungary & 51.2 \\
2 & South Korea & 44.5 \\
3 & Slovakia & 43.8 \\
4 & Norway & 42.9 \\
5 & Slovenia & 41.1 \\
6 & Denmark & 41.0 \\
7 & Portugal & 40.0 \\
8 & Barbados & 38.9 \\
8 & Japan & 38.9 \\
9 & U.S.A. & 38.6 \\
10 & Netherlands & 37.8 \\
\hline
\end{tabular}

Produced with permission: Modified from (5). incidence particularly among young adults.

\section{Epidemiology and global distribution of CRC}

CRC incidence and mortality rates vary widely across regions with the majority occurring in high-income (HDI) countries (6). The lowest rates are noted in SubSaharan Africa, except for South Africa, and Southern Asia (Figures 2,3). These global distribution and trends likely reflect changes in the prevalence of risk factors. Arnold et al. (3) noted the rise in incidence among most populations reflect lifestyle and behavioral factors including dietary patterns and obesity, whereas the mortality decline may be attributed to improvements in survival through the adoption of best practices in cancer diagnosis and treatments in developed countries.

Additionally, low and medium-income countries lack of CRC screening has significantly impacted the overall incidence and disease burden within these populations compared to high and upper-middle income countries where screening is robust. Although the risk of developing CRC increases with age, there is a unique near-global rise in incidence and mortality in early on-set CRC with a $50 \%$ increase in CRC incidence among populations younger than 50 years (Figures 4,5) (7). Epidemiologic trends predict an increase in overall crude incidence of $1 \%$ to $3 \%$ annually for adults younger than age 50 (8). This shift may represent

\begin{tabular}{|c|c|c|c|c|c|}
\hline \multicolumn{6}{|c|}{ Incidence } \\
\hline & & 2018 & & 2040 & \\
\hline & & Number & Number & Demographic change & Overall change \\
\hline World & Male & 575789 & 1014471 & $438682(+76.2 \%)$ & $438682(+76.2 \%)$ \\
\hline World & Female & 520812 & 905063 & 384251 (+73.8\%) & $384251(+73.8 \%)$ \\
\hline World & Both sexes & 1096601 & 1919534 & $822933(+75.0 \%)$ & $822933(+75.0 \%)$ \\
\hline
\end{tabular}

\begin{tabular}{|c|c|c|c|c|c|}
\hline \multicolumn{6}{|c|}{ Mortality } \\
\hline & & 2018 & & 2040 & \\
\hline & & Number & Number & Demographic change & Overall change \\
\hline World & Male & 184097 & 327212 & $143115(+77.7 \%)$ & $143115(+77.7 \%)$ \\
\hline World & Female & 126297 & 220353 & 94056 (+74.5\%) & $94056(+74.5 \%)$ \\
\hline World & Both sexes & 310394 & 547565 & 237171 (+76.4\%) & $237171(+76.4 \%)$ \\
\hline
\end{tabular}

Figure 1 Estimated number of incidence cases and deaths from CRC among both sexes and all ages; 2018-2040. Data source: Globocan 2018. Global cancer observatory. http://gco.larc.fr 
A
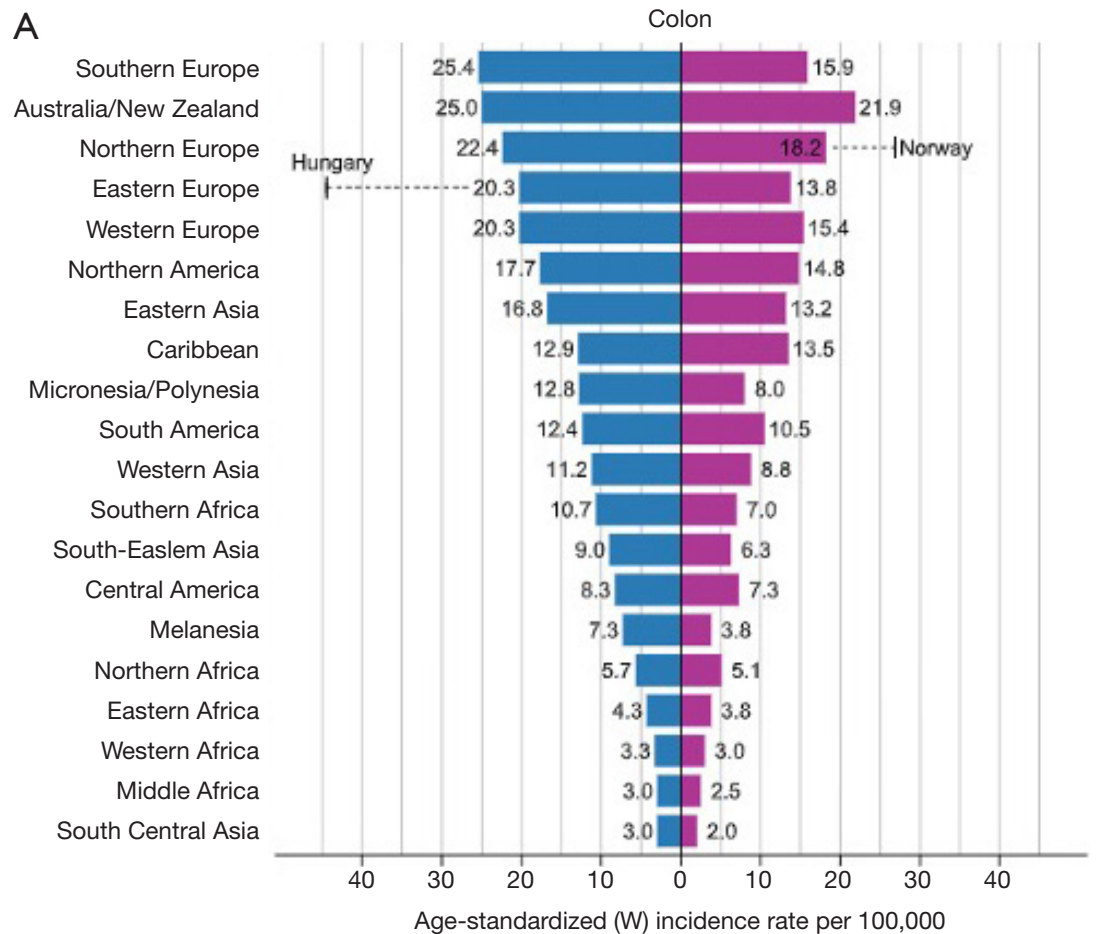

Males

$$
\text { Females }
$$

Rectum
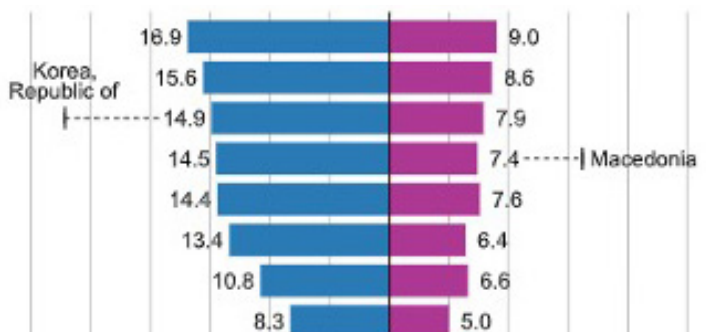

\begin{tabular}{l|l|l}
7.8 & 4.6
\end{tabular}

\begin{tabular}{l|l|l}
7.7 & 5.4
\end{tabular}

\begin{tabular}{l|l|l|}
7.6 & 5.0
\end{tabular}

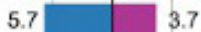

\begin{tabular}{l|l}
5.4 & 3.4
\end{tabular}

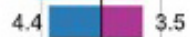

4.4

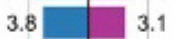

$3.7 \square$

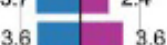

3,6

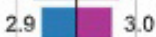

\begin{tabular}{l|l}
2.7 & 1.5
\end{tabular}

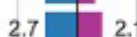

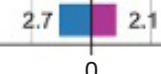

30

Age-standardized (W) incidence rate per 100,000

Males

Figure 2 Worldwide age-standardized rates for CRC are in descending order world. Bar Chart of Region-Specific Incidence AgeStandardized Rates by Sex for Cancers of the (A) Colon and (B) Rectum in 2018. Rates for cancers of the colon and rectum are shown in descending order of the world $(W)$ age-standardized rate among men, and the highest national rates among men and women are superimposed. Source: GLOBOCAN 2018. 


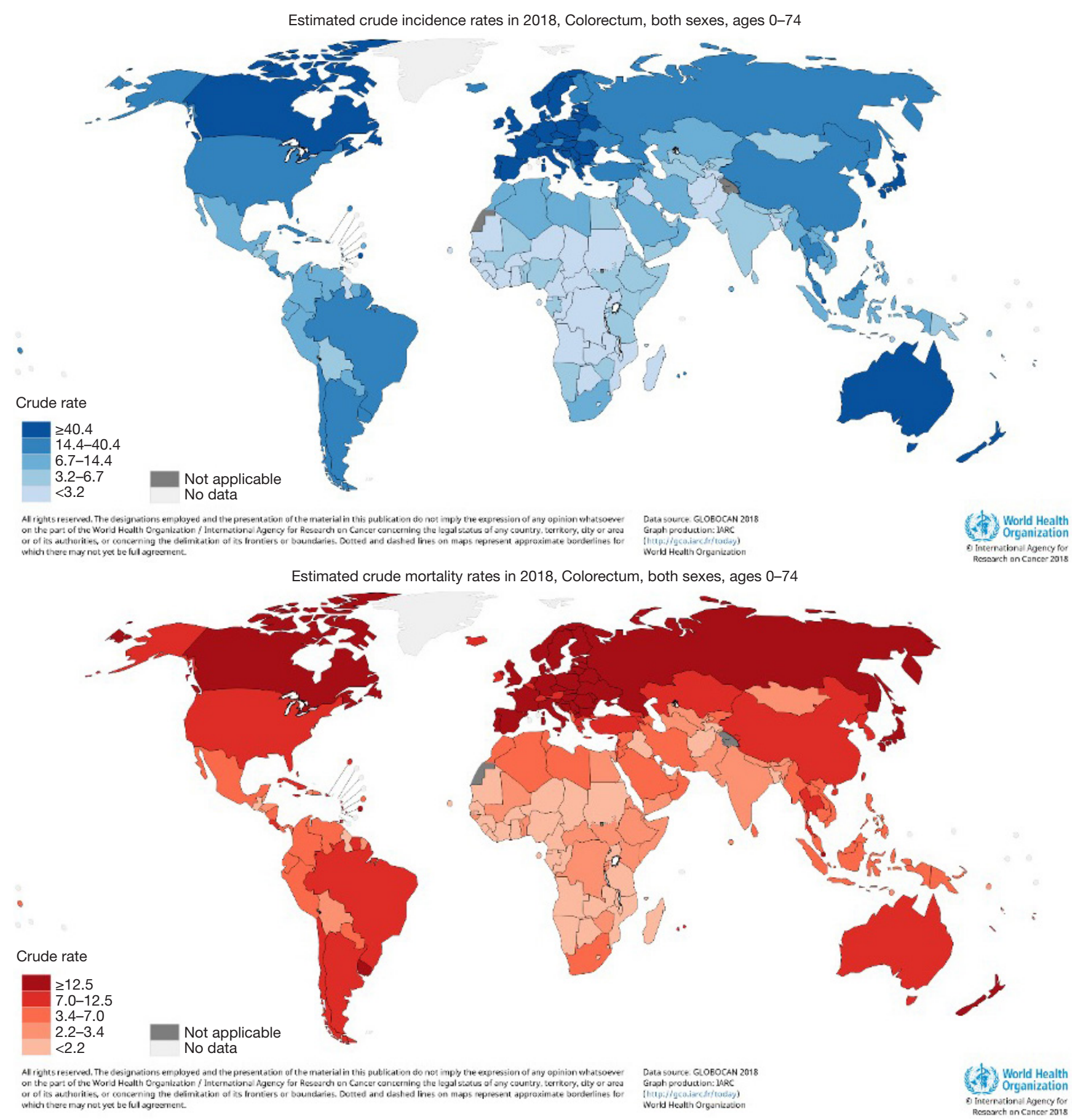

Figure 3 Worldwide estimated crude CRC incidence and mortality rates, both sexes in 2018. Source: Worldwide CRC incidence and mortality rates (age adjusted according to the world standard population, per 100 000) in both sexes in 2018 (GLOBOCAN 2018). 

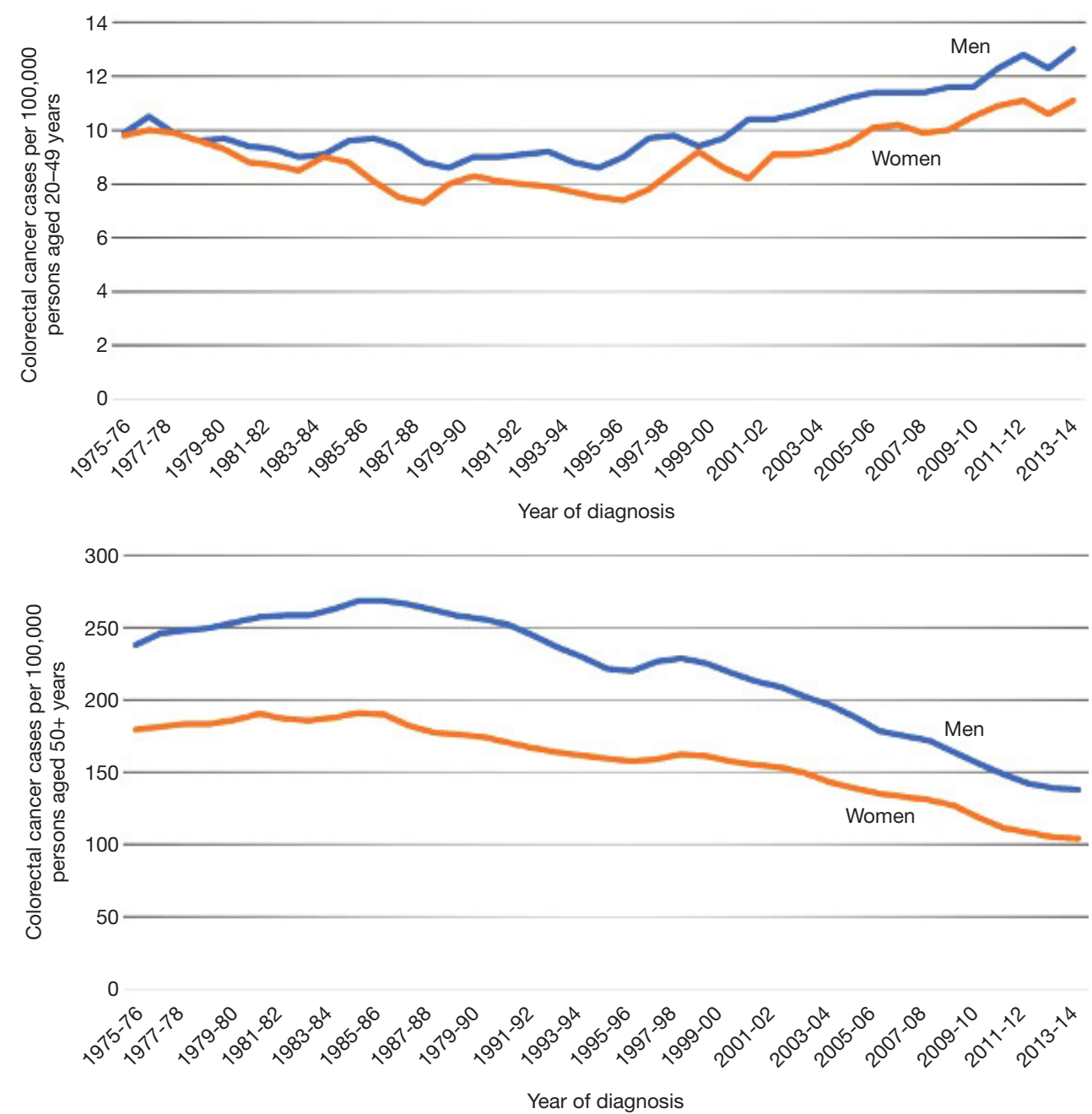

Figure 4 Trends in Colorectal Cancer Incidence (1975-2013) and Mortality (1970-2014) Rates by Age and Sex, Unite State. Data source: Surveillance, Epidemiology, and End Results program, National Cancer Institute, 2017. Rates are adjusted for delays in reporting and are plotted as a 2-year moving average.

a key to recent changes in risk factor exposures and disease pathogenesis and overall future cancer burden.

\section{Environmental influence and socioeconomic impact on CRC risk acquisition}

The gradual increase in the incidence of CRC in some low and medium HDI countries likely reflects the adoption of western lifestyles (Figure 6) (9). Among adults 50 years and older in these populations, there has been a rapid rise in the CRC incidence and mortality particularly in Eastern Europe, Asia, and South America. This is compared to very high HDI areas such as the USA, Australia, New Zealand and several
Western European where the incidence and mortality have stabilized among same cohort (10) (Figure 6). Societal and socioeconomic factors appear to affect the development of CRC. In low and medium-to-high HDI countries, increased globalization and adoption of western lifestyle with its associated CRC risk factors such as obesity, lack of exercise, tobacco and alcohol use, dietary indiscretion and pollution may be the culprit. However, the stabilization and/or decline of rates in the highest indexed HDI countries, although multifactorial, may reflect early detection through screening, both invasive and non-invasive, and advanced techniques in the management of precancerous lesions as opposed to decreased in risk factors (11-13). 


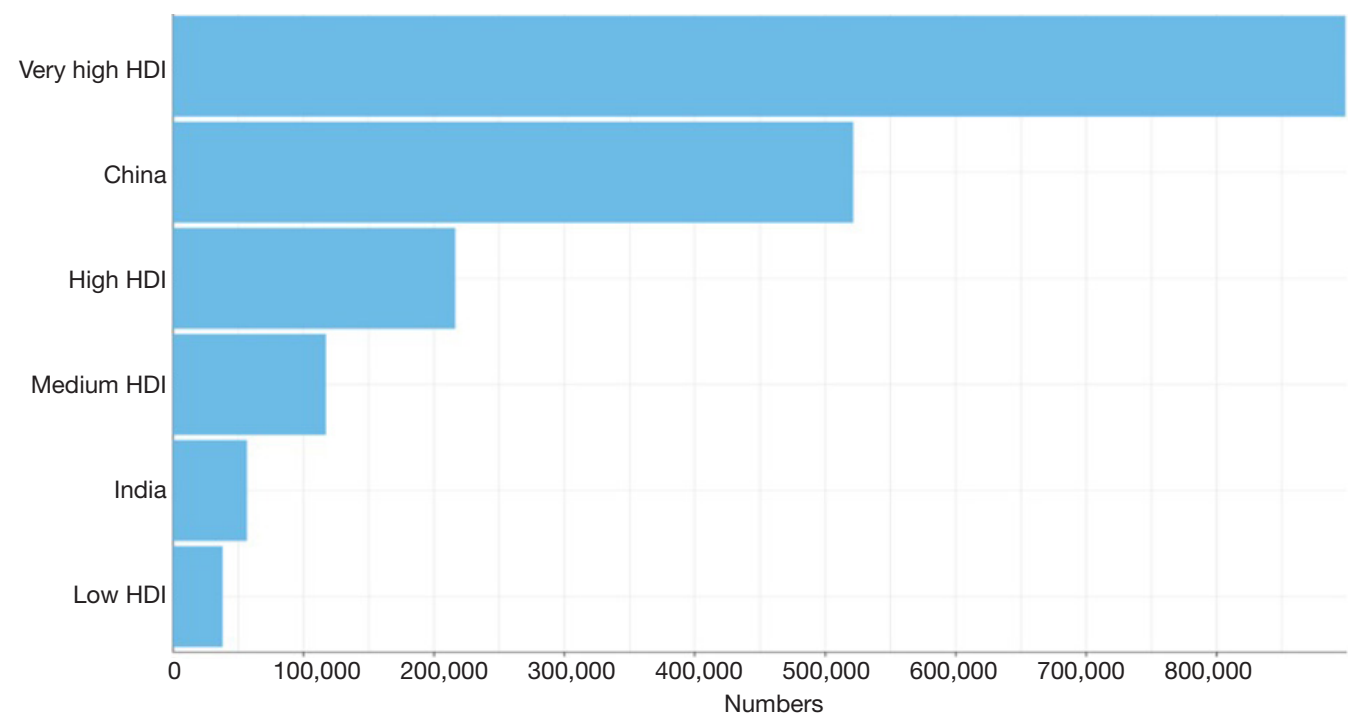

Figure 5 Estimated number of incident cases of CRC, both sexes, all ages according to country resource level. Data source: Globocan 2018. Global cancer observatory. http://gco.larc.fr
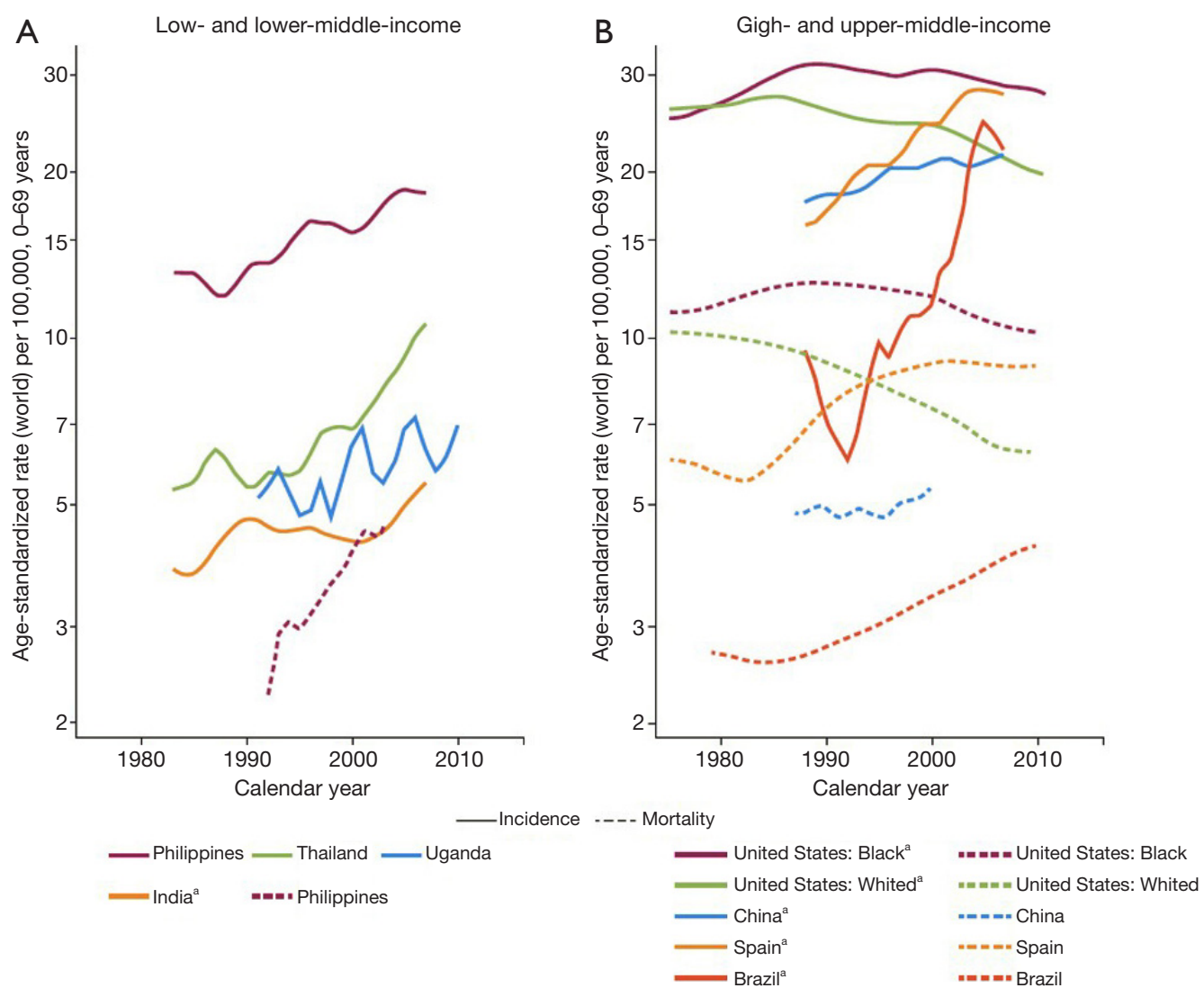

Figure 6 Trends in Age-Standardized Incidence and Mortality Rates of CRC in Men, Selected Countries by income level, 1980-2010. (Produced with permission: Bray F, Soerjomataram I. The Changing Global Burden of Cancer: Transitions in Human Development and Implications for Cancer Prevention and Control. doi: 10.1596/978-1-4648-0349-9_ch2) 
Table 2 Relative risks for established colorectal cancer risk factors

\begin{tabular}{|c|c|}
\hline Risk factors & Relative risk* \\
\hline \multicolumn{2}{|l|}{ Factors associated with increased risk } \\
\hline \multicolumn{2}{|l|}{ Hereditary and family history } \\
\hline \multicolumn{2}{|l|}{ Family history } \\
\hline \multicolumn{2}{|l|}{$\cdot \mathrm{CRC}$} \\
\hline 1 or more first-degree relatives & 2.2 \\
\hline $\begin{array}{l}1 \text { or more first-degree relatives diagnoses } \\
\text { before age } 50\end{array}$ & 3.6 \\
\hline 2 or more first-degree relatives & 4 \\
\hline 1 or more second-degree relatives & 1.7 \\
\hline \multicolumn{2}{|l|}{ - Adenoma } \\
\hline 1 or more first-degree relatives & 2 \\
\hline Inflammatory bowel disease & 1.3 \\
\hline \multicolumn{2}{|l|}{ Type 2 diabetes } \\
\hline Male & 1.4 \\
\hline Female & 1.2 \\
\hline \multicolumn{2}{|l|}{ Modifiable factors } \\
\hline - Heavy alcohol (daily average $>3$ drinks) & 1.3 \\
\hline - Obesity $\mathrm{BMI} \geq 30 \mathrm{~kg} / \mathrm{m}^{2}$ ) & 1.3 \\
\hline Colon, male & 1.5 \\
\hline Colon, female & 1.1 \\
\hline Rectum, male & 1.3 \\
\hline Rectum, female & $1.0^{ \pm}$ \\
\hline - Red meat (100 g/day) & 1.1 \\
\hline - Processed meat (50 g/day) & 1.2 \\
\hline \multicolumn{2}{|l|}{ Smoking (ever vs. never) } \\
\hline \multicolumn{2}{|l|}{ Factors associated with decreased risk: } \\
\hline Physical activity & 0.7 \\
\hline Dairy (400 g/day) & 0.9 \\
\hline
\end{tabular}

*, relative risk compares the risk of disease among people with a particular "exposure" to the risk among people without that exposure. Relative risk for dietary factors compares the highest with the lowest consumption. If the relative risk is more than 1.0, then risk is higher among exposed than unexposed persons. Relative risks less than 1.0 indicate a protective effect; ${ }^{\mathrm{t}}$, relative risk was not statistically significant. Produced with permission: Modified from (1). (C2020 American Cancer Society, Inc., Surveillance Research. CRC, colorectal cancer.

\section{Pathogenetic changes and early-life exposures that influence colorectal carcinogenesis}

There is mounting evidence to demonstrate the rising incidence and mortality of CRC in the previously perceived "lower risk" young adults. This effect has been demonstrated in both the US (14) and Canada (15) and now similar phenomenon across Europe and Asia (12). Modifiable known risk factors for CRC have been extensively investigated with previous epidemiological studies suggesting most predominantly alcohol consumption, diets high in red/processed meats, low fiber diet, obesity, smoking, and physical inactivity (Table 2). This certainly has been fueled by easy accessibility to readily available at-risk items particularly smoking and processed meats. Smoking alone in CRC will increase mortality by $23 \%$ and increase recurrence by $47 \%$. Obesity certainly parallels the increase in CRC incidence in young adults and in countries with high HDI as excess diet intake may initiate a chronic low-grade inflammatory cellular response (Figure 7). According to WHO, obesity is defined by body mass index (BMI): obesity (BMI: $30.0-34.9 \mathrm{~kg} / \mathrm{m}^{2}$ ) and morbidly obese $\left(\mathrm{BMI} \geq 40 \mathrm{~kg} / \mathrm{m}^{2}\right)$ compared to normal weight (BMI: $18.5-24.9 \mathrm{~kg} / \mathrm{m}^{2}$ ). The clinical impact of obesity on CRC appears to be more pronounced in males compared to females. Compared to normal weight individuals, the incidence of CRC has been shown to be higher in both men and women with rates of over $35 \%$ and $15 \%$ respectively (16). Weight gain and obesity appears to have a greater influence on CRC risk when it occurs in early adulthood versus later in life (17-19). Central obesity in males has been cited one of the most significant predisposing factors for CRC and worse outcome $(20,21)$.

CRC mortality is affected by obesity as high body weight measured prior to diagnosis reduces the likelihood of CRC survival. Other factors have been purported in attempt to explain the current trend. Vuik et al. proposed the low threshold for colonoscopy for diagnostic and screening purposes may have been responsible for a proportion of the detected CRCs in young adults (22). All these proposed factors remain speculative and further investigation is required to establish causation.

\section{Factors affecting CRC screening and early detection}

The CRC transition from adenoma-carcinoma is wellestablished (23). The detection and removal of premalignant 


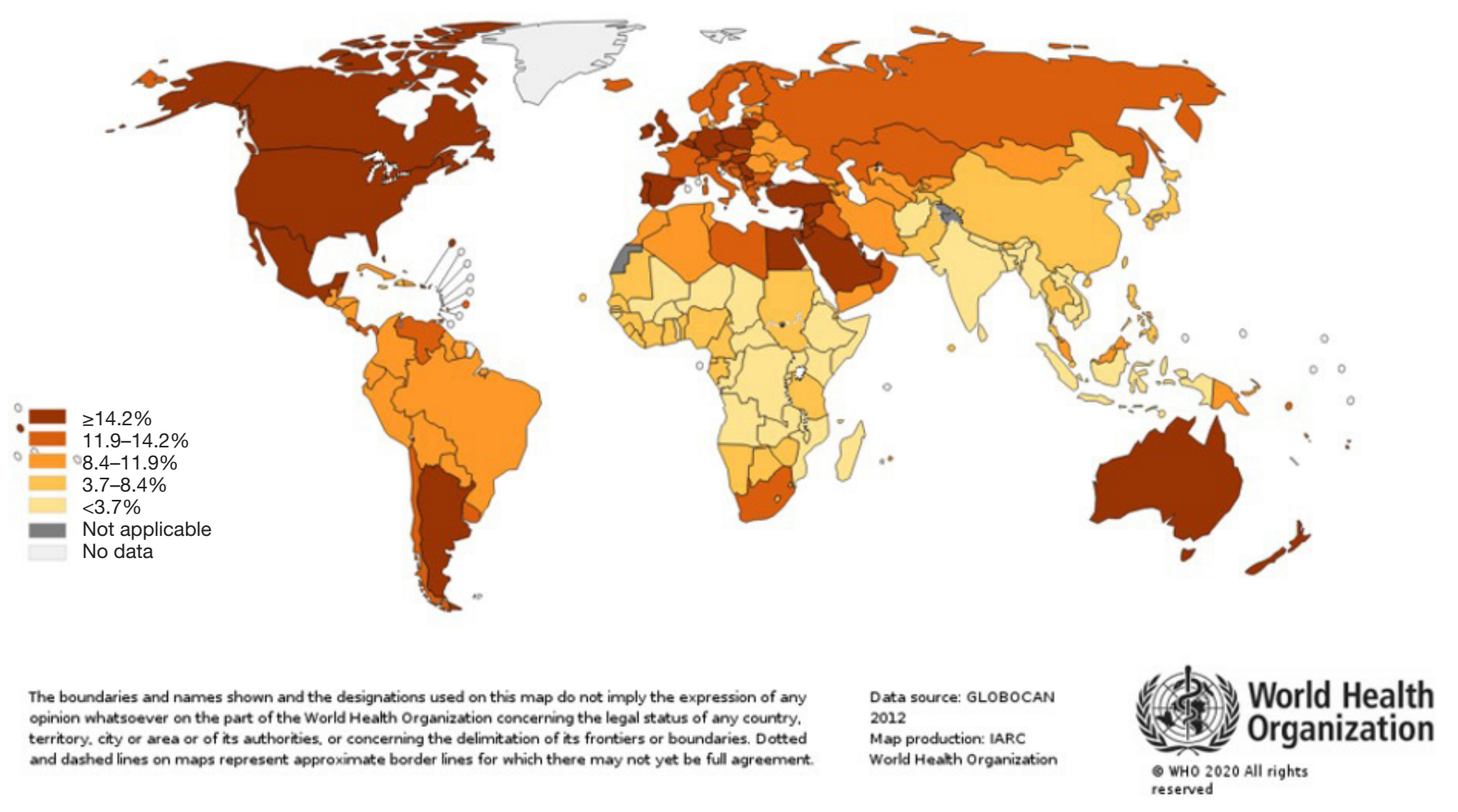

Figure 7 Fraction (\%) of all CRC cases between both sexes (worldwide) in 2012 attributable to excess body mass index.

polyps is effective in preventing and treating cancer at an early stage both by decreasing the incidence and burden of CRC. Screening methods include stools-based testing, endoscopy, and radiographic modalities with varying degree of sensitivity and specificity. For average risk population, the United State Preventive Services Task Force (USPSTF) and the American Society for Gastrointestinal Endoscopy (ASGE), and previously American Cancer Society (ACS) recommend initial screening colonoscopy (24).

The optimal screening modality is a shared decisionmaking process, which incorporates individual patient preferences, values, and risk, as well as test effectiveness, resource availability, test safety, convenience, comfort, and cost. Most strategies are based either on an annual or biennial stool-based testing (FOBTs, Fecal DNA), CT colonography every five years, with optical colonoscopy reserved for positive finding requiring further investigation, or partial/complete colonic endoscopic procedures (25). Optical colonoscopy is the most preferred and accurate of all the screening methods for CRC screening. After a wellinformed decision process, the best screening test is one that the patient is willing to complete according to the test instructions (26).

Studies evaluating the incidence of CRC following initial complete colonoscopy with polypectomy demonstrate significant reduction rates ranging from $76 \%$ to $90 \%$ $(27,28)$. The National Polyp Study demonstrated a $53 \%$ reduction in CRC-related mortality after colonoscopic polypectomy (29). The screening uptake for CRC defines the cross-sectional assessment of compliance to the screening modality and according to the ACS of the average-risk population. In March 2014, the National Colorectal Cancer Roundtable (NCCRT) launched an ambitious initiative to reach an $80 \%$ CRC screening rate of eligible adults (30). If successful, an estimated 277,000 CRC cases and 203,000 CRC deaths will be prevented by 2030 . In the US, the proportion of adults older than 50 years with a recent screening test ranged from $53 \%$ to $73 \%$ between 2011-2013 far below those of other recommended adult preventive services. Factors affecting CRC screening uptake include: sociodemographic factors, healthcare systems and provider factors, and psychosocial factors such as knowledge barriers, risk versus benefit perception concerns, and cultural biases (31). In a recent study from the ACS, Fedewa 
Table 3 Predictors of CRC non-screening rates

System-based
Availability of health insurance
Out-of-pocket expense
Availability of screening options
Provider-based
Access to a personal doctor
Specialist availability
Patient-based
Income level
Educational status
Employment status
Obesity
Race

$\mathrm{CRC}$, colorectal cancer.

and colleagues evaluated CRC screening beginning at age 45. Among people ages 45 to 49, past-year CRC screening rates rose from $4.8 \%$ in the first quarter of 2018 to $6.6 \%$ in the second quarter, $8.8 \%$ in the third quarter, and $11.7 \%$ in the fourth quarter. This was accompanied by a relatively stable rate among adults over the age of 50 (32). These factors affecting the CRC screening rate may become more relevant in the at-risk younger adult population as interventions are created to address the increasing incidence of CRC. Table 3 shows the predictors of CRC nonscreening rates with lack of health insurance often cited as a major predictor of non-screening. At a system-based level, implementing policies to decrease direct patient financial burden, increasing provider access, and standardizing screening modalities may improve on patient compliance and screening uptake. There has been tremendous progress in the field of medicine and despite the advancements in the world; there is still a lack of access to basic health needs and care. These are all issues that could be diminished by reducing the disparities that isolate these populations.

Some of these disparities are related to the geography and a shortage of physicians. In addition, income inequality with individuals and families simply unable to afford health care allows for the perpetuation of the process.

Global health professionals exploring opportunities to advocate for underrepresented communities in public health forums may solve these economic challenges. Encouraging physicians to practice in remote areas with incentives and introducing policies may reduce barriers and increase access to health care.

The USPSTF recommends the use of approved CRC screening methods in average-risk individuals. The ACS, based on its 2018 landmark population study, changed its CRC screening recommendation to 45 years of age. This recommendation has not been universally adopted particularly by the USPSTF. In the U.S., commercial health insurance and Medicare provide full coverage for the USPSTF approved screening modalities at the recommended age. However, there are some unique caveats that may affect screening colonoscopy coverage and subsequently influence out-of-pocket financial burden to the patient $(33,34)$. There has been considerable effort aimed at raising CRC screening rates (35-37). Unfortunately, these initiatives and programs are only feasible in high, and some medium-HDI countries. In 2008, the International Colorectal Cancer Screening Network (ICRCSN) conducted a survey of pilot programs with organized CRC screening programs (38). In average risk individuals, stool-based test was the primary CRC screening modality, followed by FOBT and FIT tests. This was followed by colonoscopy if any of the initial screening modality was positive. This approach may help guide the development of public health policies for the early detection of CRC even in resource-limited populations. Rabeneck et al. proposed recommendations on implementing screening and diagnosis for CRC in four different resource environments $(39,40)$ (Table 4). These recommendations, although remain to be validated, is very comprehensive and represent the basis for further research and broader implementation.

\section{Conclusions}

CRC remains one of the most preventable cancers worldwide with a clearly defined pathogenesis. However, it is a public health burden as it remains a commonly diagnosed cancer worldwide and one of the leading causes of cancer mortality. The incidence and mortality of CRC is decreasing in developed countries, while the incidence and mortality of this, based on trends, and has seen an increase in developing countries. Globally, there has been an exclusive increased in the incidence of CRC in young adults $(<50)$ as opposed to stabilization or decreased among adults over the age of 50 . The exact cause of these paradigm shifts is multifactorial. As has been noted, the higher incidence rates of CRC in countries with high HDI are associated 
Table 4 Strategies for colorectal cancer screening and diagnosis, by country resource level

\begin{tabular}{|c|c|c|}
\hline $\begin{array}{l}\text { Level of } \\
\text { resources }\end{array}$ & General principles & Diagnostic modality \\
\hline Basic, low HDI & $\begin{array}{l}\text { Capacity building: Establish } \\
\text { at risk population, primary } \\
\text { prevention strategies, create } \\
\text { cancer registry }\end{array}$ & $\begin{array}{l}\text { Barium enema if colonoscopy not available; in emergency situations, may be diagnosed at } \\
\text { surgery }\end{array}$ \\
\hline \multirow{2}{*}{$\begin{array}{l}\text { Limited, low- } \\
\text { medium HDI }\end{array}$} & $\begin{array}{l}\text { Engage in partnership } \\
\text { arrangements with cancer } \\
\text { centers to build capacity }\end{array}$ & Diagnostic colonoscopy (or barium enema) for those with symptoms \\
\hline & Establish national guidelines & \\
\hline \multirow[t]{2}{*}{$\begin{array}{l}\text { Enhanced, } \\
\text { medium-high } \\
\text { HDI }\end{array}$} & $\begin{array}{l}\text { Join international screening } \\
\text { networks }\end{array}$ & $\begin{array}{l}\text { Establish organized screening in high-incidence cities/regions starting at age } 50 \text { years in } \\
\text { persons at average risk: use annual or biennial sensitive gFOBT or FIT; FS (see text for } \\
\text { discussion of interval); or colonoscopy every } 10 \text { years }\end{array}$ \\
\hline & $\begin{array}{l}\text { Provide support to less-well- } \\
\text { resourced countries in region }\end{array}$ & $\begin{array}{l}\text { Considerable infrastructure is required to support organized screening, including } \\
\text { invitations, recalls, reminders, tracking screening test results, ensuring follow-up of those } \\
\text { with an abnormal screening test, etc. }\end{array}$ \\
\hline $\begin{array}{l}\text { Maximal, very } \\
\text { high HDI }\end{array}$ & & $\begin{array}{l}\text { National (or jurisdiction-wide) organized screening: starting at age } 50 \text { years in persons at } \\
\text { average risk: use annual or biennial sensitive gFOBT or FIT; or colonoscopy every } 10 \text { years; } \\
\text { in those at increased risk because of family history, consider colonoscopy }\end{array}$ \\
\hline
\end{tabular}

HDI, Human Development Index. Adapted from Cancer: Disease Control Priorities, Third Edition (Volume 3). Washington (DC): The International Bank for Reconstruction and Development / The World Bank; 2015 Nov 1. Chapter 6. The recommendations are meant to be cumulative: any intervention that is feasible at a lower resource level is also an option at higher resource levels (Produced with permission).

with a sedentary lifestyle, obesity, diets high in red meat, and smoking. Low mortality rates in counties with low HDI may be due to low incidence of CRC or incorrect diagnosis. Identification of independent risks factors and implementation of interventions to mitigate risk factors and increase screening uptake are paramount in the prevention of CRC. The Global correlation with the incidence of CRC and the HDI is very strong. Further work needs to continue in low HDI countries for greater access to medical care and screening for CRC.

\section{Acknowledgments}

Funding: None.

\section{Footnote}

Provenance and Peer Review: This article was commissioned by the editorial office, Fournal of Gastrointestinal Oncology for the series "Global GI Malignancies". The article has undergone external peer review.

Conflicts of Interest: All authors have completed the ICMJE uniform disclosure form (available at http://dx.doi. org/10.21037/jgo-2019-gi-06). The series "Global GI Malignancies" was commissioned by the editorial office without any funding or sponsorship. John F Gibbs served as the unpaid Guest Editor of the series and serves as an unpaid editorial board member of Fournal of Gastrointestinal Oncology from Jan 2019 to Dec 2020. The authors have no other conflicts of interest to declare.

Ethical Statement: The authors are accountable for all aspects of the work in ensuring that questions related to the accuracy or integrity of any part of the work are appropriately investigated and resolved. 
Open Access Statement: This is an Open Access article distributed in accordance with the Creative Commons Attribution-NonCommercial-NoDerivs 4.0 International License (CC BY-NC-ND 4.0), which permits the noncommercial replication and distribution of the article with the strict proviso that no changes or edits are made and the original work is properly cited (including links to both the formal publication through the relevant DOI and the license). See: https://creativecommons.org/licenses/by-nc-nd/4.0/.

\section{References}

1. American Cancer Society. Colorectal Cancer Facts \& Figures 2020-2022. Atlanta: American Cancer Society, 2020.

2. Ferlay J, Soerjomataram I, Ervik M, et al. GLOBOCAN 2012 v1.0, Cancer Incidence and Mortality Worldwide: IARC Cancer Base No. 11. Lyon, France: International Agency for Research on Cancer, 2013.

3. Arnold M, Sierra MS, Laversanne M, et al. Global patterns and trends in colorectal cancer incidence and mortality. Gut 2017;66:683-91

4. United Nations Development Programme. Human Development Report 2019: Beyond Income, Beyond Averages, Beyond Today - Inequalities in Human Development in the 21st Century. New York: UN, 2019.

5. Bray F, Ferlay J, Soerjomataram I, et al. Global cancer statistics 2018: GLOBOCAN estimates of incidence and mortality worldwide for 36 cancers in 185 countries. CA Cancer J Clin 2018;68:394-424.

6. Ferlay J, Soerjomataram I, Dikshit R, et al. Cancer incidence and mortality worldwide: sources, methods and major patterns in GLOBOCAN 2012. Int J Cancer 2015;136:E359-86.

7. Edwards BK, Ward E, Kohler BA, et al. Annual report to the nation on the status of cancer, 1975-2006, featuring colorectal cancer trends and impact of interventions (risk factors, screening, and treatment) to reduce future rates. Cancer 2010;116:544-73.

8. Center MM, Jemal A, Smith RA, et al. Worldwide variations in colorectal cancer. CA Cancer J Clin 2009;59:366-78.

9. Ansa BE, Coughlin SS, Alema-Mensah E, et al. Evaluation of colorectal cancer incidence trends in the United States (2000-2014). J Clin Med 2018;7:E22.

10. Siegel RL, Torre LA, Soerjomataram I, et al. Global patterns and trends in colorectal cancer incidence in young adults. Gut 2019;68:2179-85.

11. Gelband H, Jha P, Sankaranarayanan R, et al. Cancer:
Disease Control Priorities, Third Edition (Volume 3). Washington (DC): The International Bank for Reconstruction and Development/The World Bank, 2015.

12. Ma Y, Zhang P, Wang F, et al. Association between vitamin $\mathrm{D}$ and risk of colorectal cancer: a systematic review of prospective studies. J Clin Oncol 2011;29:3775-82.

13. Gregor MF, Hotamisligil GS. Inflammatory mechanisms in obesity. Annu Rev Immunol 2011;29:415-45.

14. Bosetti C, Rosato V, Gallus S, et al. Aspirin and cancer risk: a quantitative review to 2011. Ann Oncol 2012;23:1403-15.

15. Lin KJ, Cheung WY, Lai JY, et al. The effect of estrogen vs. combined estrogen-progestogen therapy on the risk of colorectal cancer. Int J Cancer 2012;130:419-30.

16. Ma Y, Yang Y, Wang F, et al. Obesity and risk of colorectal cancer: a systematic review of prospective studies. PLoS One 2013;8:e53916.

17. Renehan AG, Flood A, Adams KF, et al. Body mass index at different adult ages, weight change, and colorectal cancer risk in the National Institutes of Health-AARP Cohort. Am J Epidemiol 2012;176:1130-40.

18. Willett WC. Diet and cancer: an evolving picture. JAMA 2005;293:233-4.

19. Reedy J, Mitrou PN, Krebs-Smith SM, et al. Indexbased dietary patterns and risk of colorectal cancer: the NIH-AARP Diet and Health Study. Am J Epidemiol 2008;168:38-48.

20. Kormi SMA, ardehkhani S, Kerachian MA. New insights into colorectal cancer screening and early detection tests. Colorect Cancer 2015;6:63-8.

21. Bardou M, Barkun AN, Martel. Obesity and colorectal cancer. Gut 2013;62:933-47.

22. Vuik FE, Nieuwenburg SA, Bardou M, et al. Increasing incidence of colorectal cancer in young adults in Europe over the last 25 years. Gut 2019;68:1820-6.

23. Vogelstein B, Fearon ER, Hamilton SR, et al. Genetic alterations during colorectal-tumor development. N Engl J Med 1988;319:525-32.

24. Wolf AMD, Fontham ETH, Church TR, et al. Colorectal cancer screening for average-risk adults: 2018 guideline update from the American Cancer Society. CA Cancer J Clin 2018;68:250-81.

25. American Cancer Society. Colorectal Cancer Facts \& Figures 2017-2019. Atlanta: American Cancer Society, 2017.

26. Bibbins-Domingo K, Grossman DC, Curry SJ, et al. Screening for Colorectal Cancer: US Preventive Services Task Force Recommendation Statement.US Preventive Services Task Force, JAMA 2016;315:2564-75.

27. Zauber AG, Winawer SJ, O'Brien MJ, et al. Colonoscopic 
polypectomy and long-term prevention of colorectalcancer deaths. N Engl J Med 2012;366:687-96.

28. Citarda F, Tomaselli G, Capocaccia R, et al. Efficacy in standard clinical practice of colonoscopic polypectomy in reducing colorectal cancer incidence. Gut 2001;48:812-5.

29. Winawer SJ, Zauber AG, Ho MN, et al. Prevention of colorectal cancer by colonoscopic polypectomy. The National Polyp Study Workgroup. N Engl J Med 1993;329:1977-81.

30. Meester RG, Doubeni CA, Zauber AG, et al. Public health impact of achieving $80 \%$ colorectal cancer screening rates in the United States by 2018. Cancer 2015;121:2281-5.

31. Gimeno García AZ. Factors influencing colorectal cancer screening participation. Gastroenterol Res Pract 2012;2012:483417.

32. Fedewa SA, Siegel RL, Goding Sauer A, et al. Colorectal cancer screening patterns after the American Cancer Society's recommendation to initiate screening at age 45 years. Cancer 2020;126:1351-3.

33. Selby JV, Fireman BH, Swain BEN. Effect of a copayment on use of the emergency department in a health maintenance organization. N Engl J Med 1996;334:635-41.

34. Ladabaum U, Levin Z, Mannalithara A, et al. Colorectal testing utilization and payments in a large cohort of commercially insured US adults. Am J Gastroenterol 2014;109:1513-25.

35. Baker DW, Brown T, Buchanan DR, Weil J, et al. Comparative effectiveness of a multifaceted intervention to improve adherence to annual colorectal cancer screening in community health centers: a randomized clinical trial. JAMA Intern Med 2014;174:1235-41.

36. Dietrich AJ, Tobin JN, Robinson CM, et al. Telephone outreach to increase colon cancer screening in medicaid managed care organizations: a randomized controlled trial. Ann Fam Med 2013;11:335-43.

37. Murphy CC, Vernon SW, Haddock NM, et al. Longitudinal predictors of colorectal cancer screening among participants in a randomized controlled trial. Prev Med 2014;66:123-30.

38. Benson VS, Atkin WS, Green J, et al. Toward Standardizing and Reporting Colorectal Cancer Screening Indicators on an International Level: The International Colorectal Cancer Screening Network. Int J Cancer 2012;130:2961-73.

39. Rabeneck L, Horton S, Zauber AG, et al. Colorectal Cancer. In: Gelband H, Jha P, Sankaranarayanan R, et al. editors. Cancer: Disease Control Priorities, Third Edition (Volume 3). Washington (DC): The International Bank for Reconstruction and Development/The World Bank, 2015.

40. Bray F, Soerjomataram I. The Changing Global Burden of Cancer: Transitions in Human Development and Implications for Cancer Prevention and Control. In: Gelband H, Jha P, Sankaranarayanan R, et al., editors. Cancer: Disease Control Priorities, Third Edition (Volume 3). Washington (DC): The International Bank for Reconstruction and Development/The World Bank, 2015.

Cite this article as: Vabi BW, Gibbs JF, Parker GS. Implications of the growing incidence of global colorectal cancer. J Gastrointest Oncol 2021;12(Suppl 2):S387-S398. doi: 10.21037/jgo-2019-gi-06 